\title{
Mobile Health (mHealth): A Conceptual View
}

\author{
Sergey Pankratov ${ }^{1, *}$, Tatiana Znamenskaya ${ }^{2}$ \\ ${ }^{1}$ Technische Universität München, Computer Science Dept (Informatik-5), Boltzmannstr. 3, D-85748, Garching, Germany \\ ${ }^{2}$ Microsoft Deutschland GmbH, Konrad-Zuse-Str. 1, D-85716, Unterschleissheim, Germany \\ *Corresponding Author: Sergey.Pankratov@online.de
}

Copyright (C) 2014 Horizon Research Publishing All rights reserved.

\begin{abstract}
The value of mHealth for sustainable healthcare is discussed. It is projected that mHealth can become a disruptive technology that is set out to radically transform the current state of medical disciplines. In particular, the mHealth solutions can provide better understanding of the organism stability margins and of the emergence of diseases as well as ensure more patient safety under drug and physiotherapy prescription. The issue of measuring the physiological quantities is addressed, with the conventional yet complex task of quantitative accuracy evaluation being discussed. The concept of complexity and its relevance for the physiological parameters defining both the health state and disorders is accounted for. The principles of body area networking and the relevant standards are briefly overviewed, with a focus on security issues. The concept of biofeedback implemented through mHealth sensor technologies is examined. Basic requirements to facilitate market acceptance and response are analyzed. Selected case studies of pervasive health monitoring are presented. Possible current and future mHealth applications are considered. Since some general problems of healthcare are touched upon in the article, it is intended to provoke a controversy.
\end{abstract}

Keywords Homeostasis, Sensor, Networking, Measurement, Biofeedback

\section{Introduction}

The notion of Mobile Health (mHealth) is much wider than just the usage of wearable medical devices such as, e.g., 24-hours blood pressure monitors. Mobile Health offers a different paradigm of healthcare as compared to today's mainstream medicine. Furthermore, mHealth fosters the development of non-intrusive sensor-based techniques of picking up parallel information from multiple body areas in real time, thus measuring the distributed state of human health. Fresh ideas and novel engineering approaches induced by the development of mHealth can change the concept of healthcare and influence the worldview both of medical specialists and of increasing number of professionals dealing with the medical branch, e.g., software developers.

Although the notions of mHealth and telemedicine are often used interchangeably, they are not completely identical. In these two new disciplines, accents are put on different functionalities and technological areas: mHealth is mainly focused on taking up physiological parameters of a functioning organism (with or without any pathological process or the organism's instability) whereas telemedicine mostly deals with remote physician's consultations or, in exceptional cases, distant therapies. Accordingly, the underlying technologies have non-intersecting components: while mHealth uses a combined or even networked system of more and more miniature sensors placed on a human body (WBAN), with the intention to produce non-demolition measurements or registration of health parameters, telemedicine may freely utilize highly invasive (e.g., biochemical) techniques, stationary lab investigations/therapies such as X-rays, CT, MRI, PET screening, pulsed lasers, proton therapy or other procedures based on clumsy devices excluding any mobility or non-invasive monitoring. Note also that the state of an organism can be significantly changed or even destroyed by observation. The focus of telemedicine is to ensure a sustainable telecommunication between the entire healthcare matrix: patients, doctors, clinics, insurance companies, governmental health organizations, etc. In other words, the main point in the telemedicine is the remote access to data, while the data flow in mHealth is predominantly restrained to the mobile communication (presumably in the automatic mode) of a patient or a healthy person with her/his health provider. Nonetheless, there are of course many overlapping areas in mHealth and telemedicine.

There are two core virtues related to mHealth. Firstly, mHealth is capable of ubiquitous healthcare delivery thus turning medicine into health maintenance. Secondly, mHealth would require the development of novel technologies and advanced engineering solutions, as concerns primarily hardware miniaturization and creation of dedicated software applications. The economic factor is also important: high costs of any contact with a doctor are drastically reduced and the precious time of highly qualified medical personnel is saved. Accordingly, the concept of 
distributed healthcare as health maintenance system and new technological solutions supporting this concept are discussed in this paper.

\section{The State of Health}

It is indicative that one talks about Mobile Health, not Mobile Medicine. Apart from an obvious convenience of the consonant pronunciation (mHealth), there is a deep meaning in that. Although the notions "health" and "medicine" are commonly used interchangeably in various contexts, there is a crucial difference between them since medicine deals with deviations from healthy conditions and treats diseases, mostly by medications, surgery and, since comparatively recent times, by laser and ionizing radiation. The very term "medicine" stems from the ancient "art of healing" i.e. returning to health. It is not accidentally that the object of treatment in medicine is known as "patient" whereas health can (and should) be maintained in any person.

In connection with mHealth, it may be vital to formulate the notion of being healthy. People feel themselves healthy as long as their organisms are capable to maintain stability under an influx of external real-life influences such as stress, psychic overload, microorganisms, fungi, allergens, parasites, etc. Notice that health is a holistic notion: there is no such thing as a partial health. Conversely, if all body organs are functioning "normally" i.e. they can be found by certified medical experts in a state within the respective physiological norms, it does not preclude the existence of malfunction on the level of entire organism. This fact is typical of complex systems: the whole is not the sum of its parts. The notion of the holistic nature of health state of course does not contradict to analytical evidence-based medicine and is not necessarily equivalent to the concepts existing in oriental medical alternatives.

The mainstream medicine, being split among a number of specialized disciplines devoted to the treatment of diverse diseases, does not handle the healthcare state on an adequate level. Accordingly, the preventive healthcare has not yet become a science i.e. no regular, measurable and reproducible techniques have been elaborated to monitor the sustainable body homeostasis and deviations from it. Medicine as an applied scientific discipline tends to produce a strange impression on a person with engineering background or trained in natural sciences, primarily because the results of measurements (if at all obtained) are often presented without giving error bars. Medical diagnostics is basically a technique of intuitive feature extraction, combining fuzzy attributes into a plausible pattern. Accurate diagnosis, even based on currently available precise instrumentation, can take years. No reliable evaluation of medical diagnoses, nor efficient algorithms for diagnostics appear to be known (see, however, [1,2]). Most conclusions about the state of a patient are more descriptions rather than actual diagnoses. Medical specialists usually refer to intuition or impression to discern the basic (irreducible) physiological characteristics, both in health state and in that of functional disorder. Most of healthcare professionals, especially in the developing countries, are still inclined to oppose number and understanding.

Why has not the health state been thoroughly described in medicine? At the first sight, it should not be that complex: just take some appropriate physiological features, assign the "coordinate axes" (to provide, e.g., a numerical representation) and partition the feature space embracing all physiological attributes into subdomains (classes). This procedure would provide the means to recognize health areas and bring at least a full phenomenological classification - the initial stage in any hard science. Moreover, both health areas and their borders could then be depicted as objects in object-oriented programming techniques. The fundamental trouble, however, is that the dimensionality of the physiological state space is so huge that no workable mathematical classification seems to produce practically useful results, at least so far. Recall that the blood test alone counts over 30 independent variables. Neither statistical approaches nor fuzzy sets help much to provide an essential description of health. Therefore, instead of the general description of the functioning organism one has to resort to an $a d$ hoc and mostly subjective selection of physiological features, e.g., skin color, gait, BMI (body mass index), pulse rate, blood pressure, etc. One might call such standardized observations "low-tech" means of gathering physiological information.

Contemporary approaches to mental health are specifically indicative: they are basically "low-tech" and highly subjective. The data relevant to mental health assessment are contaminated with cognitive and perceptual biases of the assessing person. There exist a number of classification schemes, but their applicability limits seem to be totally unclear in the scientific (measurement-based) sense. For example, the functional disorders which are loosely described as stress, burn-out syndrome, depression and the like do not have adequate representation in operational (quantifiable) terms. The body states with the focus on "consciousness" described in such disciplines as psychology, psychoanalysis, psychiatry and the related areas more rely on speculative opinions and metaphors (often of archaic character) rather than on objective and reproducible characteristics endowed with quantifiable error margins. However, powerful pharmacological agents administered on plainly intuitive grounds disguised as the best available science can readily drive a person onto an unstable trajectory. A related question is: how can one assess whether a therapist is effective or not?

It is significant that the concept of norm (i.e. that of mental health) in behavioral disciplines is rather hard to establish. Such states as autism or schizophrenia are vaguely classified through behavior signs, without any delimitation of observed (in particular, physically observed) quantities. Recent attempts of experimental validation of the psychotherapeutic treatment using PET (positron emission tomography), SPECT (single photon emission computer tomography), 
MEG (magnetoencephalography) and nuclear spin tomography (e.g., fMRI or MSI - magnetic source imaging) have so far only the research status, are prohibitively expensive and met with skepticism by actively practicing medical professionals. Notice, however, that there appears to be a growing interest in wearable computers and sensor deployment in behavioral disciplines. Yet objective measurements and in general quantitative approaches still are not perceived within the value-based mental health community as a viable option for healthcare (see, e.g., [3]).

Notice that nearly all contemporary physics-based techniques require heavyweight equipment and specially trained personnel. For instance, MEG allowing one to visualize the neuron activity inside the brain with good spatial resolution in nearly real time (temporal resolution about $10^{-4} \mathrm{~s}$, spatial resolution approximately $1-5 \mathrm{~mm}$ ) currently requires magnetically isolated laboratories that a rare hospital can afford. With the development of sensor technologies, such complicated and expensive installations possibly can be replaced by multiple light-weight sensors/actuators controlled by clever signal processing, inverse source detection and noise cancellation software. Appropriate sensors might be, e.g., SQUID (superconducting quantum interference devices) or spin-exchange magnetometer (SERF) arrays, provided of course that suitable HTSC (high-temperature superconductive) materials, technologically expedient and commercially attractive can be attained. Notice that contrary to EEG (electroencephalography) that mainly registers the neuron activity on the surface of the cerebral cortex, giving little information about subcortical neuron activity so that clinicians have to extrapolate the picture, MEG is capable of register the signals produced by the currents excited in subcortical areas, thus providing $3 \mathrm{~d}$ information.

Linking the novel sensor-based mHealth technologies to the existing descriptive physiology can provide a reliable empirical basis for the development of the health science. At least a number of models describing the body as a functional macrosystem interacting with the environment can be produced based on the data obtained from the mHealth solutions monitoring the organism under "normal" conditions i.e. unperturbed by a medical intervention. Deviations from the health state can be transient, with the body quickly (in the time-scale of a few hours) returning to the stable mode, or long-term (over several days) and runaway states i.e. corresponding to a progressive disease with the development of possible life-threatening physiological anomalies. Using the fashionable today language of synergetic and dynamical systems theory, one can say that state of health is an attractor for the human body. (Synergetic is a loosely defined interdisciplinary field, where cooperative processes of self-organization in complex systems are studied; dynamical systems are those that undergo temporal evolution.)

Despite all the achievements of medical science, both the state of health and deviations from it labeled as diseases, ailments, illnesses, pathologies, disorders, dysfunctions, disabilities, anomalies and so forth are still suffering from the lack of objective evidence. The sheer number of available synonyms implies diagnostic uncertainty and the possibility of misconceptions. It would be important to quantitatively establish health robustness which is the notion related to the stability of the whole organism and not to any of its subsystem (organ). The robust state of health does not significantly change when disturbed by not very strong external influence - otherwise such activities as, e.g., sports would frequently involve dramatic consequences. Contrariwise, the organism outside of the health domain is unstable (or even structurally unstable). Health domain tends to shrink with age and can be affected by certain chronic conditions such as diabetes, cancer, HIV, etc.

Without any attempt to belittle the doctors' merits one has to admit that the impressive achievements of contemporary medicine are mostly due to explosive development of high-precision digital instrumentation i.e. applied physics, semiconductor engineering and software development. Physiological status of a body is still described in intuitive terms that usually admit non-unique interpretations. Furthermore, contemporary medicine mainly relies on averaged statistical data i.e. individual characteristics are largely neglected (being habitually labeled as "unspecific"). This approach appears to be methodologically wrong since it is based on an implicit assumption of the Gauss distribution underlying medical statistics. Yet the statistics of emergent diseases, similarly to heavy accident statistics, seem to obey power law distributions rather than exponential ones. Note that statistical results, e.g. produced in clinical trials, in fact do not answer the crucial question: what is the best strategy for a given patient.

The vast amount of experience accumulated in various medical disciplines busy with specific disease treatment can be used as a set of limiting cases corresponding to excessive body reactions to the real-life excitations and impacts (e.g., of physical, chemical or biological character). Each person possibly has a natural (inborn or developed) health margins within which the body does not develop excessive reactions leading to functional disorders described as pathological conditions. Mathematically speaking, the health state is a manifold in the space of all possible physiological parameters. The development of sensor-based mHealth systems can provide the health margins as quasi-stable individual characteristics (like body size, face geometry or the sleep duration need for each individual). Sensor-based body networking can also provision non-invasive early diagnostics pertaining to the cases when certain body parameters get outside of the health margins. Moreover, besides diagnostics sensor-based mHealth systems can offer some prefatory means for fixing body problems, e.g., through biofeedback mechanisms inducing the organism's natural self-regulatory reactions that might restore its functional equilibrium.

Humans, as a rule (unless properly trained), can only poorly control their physiological parameters, therefore most subsystems of the human organism are functioning in the 
autonomous mode. The primary role of biofeedback is to correct errors, to damp large fluctuations and to retain the health state. The body sensors control the health parameters, while the latter, in their own right, affect the functioning of body sensors, thus achieving a self-consistent state of equilibrium. Biofeedback channels connect the observed physiological output with the intrinsic signal sources, usually deeply hidden inside of CNS (central nervous system) i.e. brain or spinal cord. Assume that a given sensor or a group of sensors have registered the values that are deviating from the health domain. Then a person can purposefully act - possibly with the aid of other people, e.g., medical professionals - in order to regain the health state, for instance, by medication intake or through proper exercising (physiotherapy). This action would be a prototypic example of a biofeedback loop involving the mediation of human consciousness (individual or collective). Biofeedback can also be automatically activated, e.g., via commensurate signals put on mHealth actuators.

One might notice that the oriental health maintaining systems have also - just as the European medicine accumulated massive experience in dealing with human organism, but the methodology has been quite different. Oriental systems considered the body as a homeostatic system that is capable to mobilize latent resources to stay within the domain of health or return to it when driven from stability. It is a pity that the wide-spread concepts of oriental health maintenance systems do not seem to have been adequately and objectively studied using modern technologies and highly sensitive measuring devices (see in this respect [4]).

The matter is that, contrary to the dominant - and unverifiable - presumption that diseases mainly arise from erroneous behavior or unreliable functioning of individual body organs, partly genetically determined, the mHealth paradigm leads to the concept that diseases are rooted in the very foundation of the complexity of a human organism that involves numerous interconnected components and subsystems. The possibility of a disease does not spring from the failure of a distinct body part (organ), although it can be manifested as such, but is the property of the whole organism. Metaphorically speaking, if you have a child, the chances are that she/he may have measles. For a complex system with a strong coupling between its components, a new factor appears compared to a set of individual elements, which is a consequence of extremely complicated - multidimensional and entangled - organism dynamics. This new factor i.e. collective behavior can lead to poorly predictable dependence of the final state of the organism on its initial state. Thus, nearby initial states do not necessarily imply close organism's behavior all time in the future, e.g., two initially similar states can develop into completely different ones. As a result a serious disease can occur unexpectedly if it is compatible with the possible organism dynamics. Notice that emergence of an unexpected or unexplained pathology tends to be interpreted as accidental or sometimes as "fate", although in fact a small fluctuation, minor functional defect or age-related unevenness can push the organism to the path leading to a disease.

The human organism can be regarded as a complex open system in a state of delicate equilibrium exchanging chemical substances, energy and entropy (information) with the outer world. Since the whole organism consists of a number of strongly coupled and cohesively working subsystems the failure of any one of them tends to provoke an avalanche of other failures. Therefore, treatment of a single organ or a separate disease usually leads to complications involving other organs - an effect well-known to any practicing doctor. The contemporary mainstream medicine is built on dissecting the entire homeostatic system of human body into diseases that can be grouped to form a medical specialty. Each medical professional is focused on repairing a certain part of the body without paying much attention to the integral body status. Such an approach inevitably leads to more and more radical interventions favored also by economic considerations. The standard policy of medical professionals is to resort to radical intervention as soon as possible, often without sufficient reasons. Yet radical treatment strains the natural functionality of the organism and can drive it out of the inherent health (stability) margins, which unfortunately happens quite often even in the developed countries. This fact is reflected in numerous cases of worsened patient conditions following radical medical treatment. Hundreds of thousand patients die each year (that equals to the population of a large city) of the consequences of medical treatment, such deaths being largely interpreted as "medical errors" or even as "fate". Of course much grief, unnecessary costs, time-consuming professional procedures and litigation ensue. Accordingly, the risk of disability or death due to medical treatment is at least several times higher than the same risk due to car accident and exceeds by many orders of magnitude the respective risk due to severe accidents with nuclear power plants.

When an external influence i.e. the "signal" strength (e.g., temperature, pressure, concentration of a chemical substance, etc.) approaches the boarders of stability, the state becomes unstable and a transition to a new state (that of illness) occurs. Usually in the critical region near the health borders large fluctuations of the body parameters such as body/blood temperature, arterial pressure, heart rate, oxygen concentration, skin wetness, etc. can be observed which are often interpreted as manifestations of a "crisis". Note that crisis is a normal process in any complex (highly nonlinear) dynamic system. One can, in principle, damp crises by introducing or increasing some equivalent of dissipation in the system (effective dissipation), but this measure can lead to some stationary regime of stagnation. In medicine that can be regarded as a specific case of controlling a complex system (human organism) damping is tantamount to carrying out a desensibilizing therapy. Suppression of acute crisis symptoms such as pain, cramps or neurological disorders by strong medications can lead to the drastic suppression of normal physiological functions manifested, e.g., as 
hyporeflexia and various autonomic dysfunctions.

Transitions from health to disease caused by fluctuations mostly reflecting the influence of the surroundings may be considered a special case of a general phenomenon of noise-induced transitions from a more ordered (low-entropy) to a more disordered (increased entropy) state in a complex system interacting with the environment. Note that fluctuations and noise leading to stability (health) loss can be both external and internal. In simple words, an organism is always subjected to perturbations related to the unavoidable emergence of errors. The crucial question is whether one can keep such errors under control, thus maintaining the health state, or the errors will be accumulated and amplified in the organism evolving in time, in which case the disease (instability) would develop.

\section{Limits of Stability and the Role of Medicine}

The mHealth enables to monitor physiological reactions of the whole organism under natural real-life conditions, with no additional stress or health perturbations associated with many standard medical procedures that can aggravate the patient condition. For example, the ubiquitous exercise-electrocardiogram (stress ECG or cardiac stress treadmills) is favored by the doctors but can be contingently detrimental for the patients. Other habitual yet adverse effects involve quick drug prescriptions: there are numerous medications (for example, those used to treat metabolic diseases) that can be highly toxic or even lethal after an inadvertent overdosing. Moreover, quite often the doctors do not even think of warning the patients that they should not drive a car or operate mechanisms while taking medications, even seemingly innocent ones - with high and unknown risk of an accident. Side effects of drugs, their interaction in a specific organism as well as overmedication are not sufficiently explored. Furthermore, slowly growing concentrations of seemingly innocent medications in the body can reach a critical point in which the health state loses stability and a serious pathology can develop (one might recall in this connection the untimely death of Michael Jackson, one of the most prominent music superstars, of a combination of prescribed sedatives).

The same applies to the "accident concentration" i.e. gradual accumulation of unpleasant events. For example, any event challenging human adaptive mechanisms such as nervous tension, lack of time, failure, humiliation, scornful attitude, sneer, feeling of helplessness, etc. provokes stress, and as such events can rather frequently occur in modern urban environment, many million people suffer from unnecessary and, what is worse, uncontrollable stress that may cause a serious health hazard.

In everyday reality, radical strategies in medicine such as extensive use of active chemical substances and/or physiological procedures quite easily provoke conflicts with various systems in human organism, the latter always striving to maintain its homeostasis. The latter is ensured by maintaining and monitoring physiological variables within certain stability limits. Physiological variables such as those that can be obtained by a blood or urine tests (concentrations of glucose, hemoglobin, cholesterol, triglyceride, creatinine, albumin, ions, bilirubin, red blood cell distribution width $\mathrm{RDW}$, etc.) describe an internal state of the organism. In medicine, one often talks about the internal environment. Maintaining an organism within the homeostatic stability limits is mainly effected through negative feedback mechanisms involving the endocrine and the nervous systems.

A rather primitive illustration of health (stability) margins is the ubiquitous reaction of the human organism to alcohol (ethanol). Small doses of this substance i.e. blood alcohol concentration (BAC) less than 30 milligram of ethanol in 100 millilitres $(0.11)$ of blood or 0.3 promille $(0.3 \mathrm{~g} / \mathrm{l})$ do not appear to seriously impair cognitive abilities or locomotion of an "average" person. This value might be roughly taken as the boundary of the stability (health) domain. Returning to this stability domain occurs with the estimated rate of 0.15 promille/hour, although of course this relaxation process may vary from person to person. Both the health state and the restoring rate depend on other variables such as age, gender, BMI, etc., not only on BAC, which illustrates the fact that the health (stability) domain and the restoring path cannot be uniquely defined for all persons and conditions. In general, most chemical substances in the body have their specific threshold levels with respect to their concentration in blood.

One cannot avoid failures in physiological subsystems of a functioning organism, as in any complex system, e.g., in technology or society. Therefore, one needs to explore both functional and physiological limits both of such subsystems and of the organism as a whole. Quantitatively exploring the limits of stability i.e. those of health would greatly contribute into the understanding of how, why and under what conditions the organism may fail to function properly. Many medical professionals seem to understand the problem of finding the boundaries of the health domain, but try to establish them by habitually crude methods verging on the loss of stability. One can recall the Nazi medical experimentation on humans intended to test the limits of stability of the functioning organism under, e.g., freezing or elevated environmental temperatures and extremely high or low air pressures. Many prisoners of war and Jews were subjected by the Nazi doctors to highly virulent infections, poisonous gases and starvation. Similar experiments were carried out by the Japanese military doctors, especially in China. However, not only under war conditions, but also in peaceful times, medical research accompanied with deliberate harm (i.e. breach of stability) inflicted to human subjects was performed in a number of countries claiming to have well-developed healthcare services. For instance, in autumn 2010, the US Secretary of State Hilary Clinton officially apologized for the experiments performed by the US Public Health Service on unsuspecting patients in Guatemala who were deliberately infected by syphilis (see, 
e.g., [5]). History of controversial medical experiments involves harmful surgeries performed by J.M. Sims (known as the "father of gynecology") on slave women [6].

Even today, some dubious medical techniques driving the patient outside of the health stability domain (such as, e.g., trepanation, electroconvulsive therapy and lobotomy) can be occasionally administered. All these techniques are highly invasive; they are crude surgical or physical procedures on the functioning brain considered fully justified to treat some vaguely described disorders. Actually, trepanation is an ancient method consisting in drilling holes in the patient's skull (typically with no anesthesia). Trepanation was (and continues to be) justified as a means of relieving intracranial pressure; although there are serious doubts that this old and dangerous practice is a valid medical procedure to treat such disorders as migraines and occasional brain seizures. Trepanation is akin to an ancient Greek belief that letting the body humors out (a well-known example is blood-letting) would remove all imbalances in the organism and thus cure all diseases.

Trepanation may compete as regards its invasivity and high potential for irreparable damage with another brute force procedure known as lobotomy whose original form was also based on drilling holes in a person's skull. It is interesting that lobotomy initially gained a lot of accolades from the medical and psychiatric communities. The inventor of this brain damaging technique (lobotomy consists in deliberately destroying the brain connections), the Portuguese neurologist Egas Moniz, was awarded the 1949 Nobel Prize in Medicine. Although Moniz himself was an outstanding person, this Nobel Prize clearly demonstrated a complete disregard of the patients' interests within the medical community. Inspired by Moniz, an arduous popularizer of lobotomy, Dr. W. Freeman, was known for lobotomizing with the help of ice picks and mallet (together with his neurosurgical colleague J.Watts) over 2500 persons in the USA alone; Freeman also reportedly used an electroshock to immobilize his patients. Practically all those patients turned into dull, passive, meek, vacant and dependent creatures without any initiative or spontaneity, nearly all reportedly developed incontinence and some died of a brain hemorrhage. Notice that such a submissive personality corresponds to the traditional concept of an ideal patient as viewed by the medical community: an obedient object allowing any doctor to be acted upon instead of taking active role in her/his care. Lobotomy basically consists in severing the connecting fibers between the prefrontal cortex and other parts of the brain, in particular, cutting through the corpus callosum which is a gateway between the left and right cerebral hemisphere. Over 100000 persons are estimated to have been lobotomized in the developed countries, before this method was abandoned in 1970s [7]. Yet highly destructive technique of lobotomy became an accepted medical procedure, and one can still undergo it today, yet under more professionally sounding names of a corpus callosotomy or lobectomy [8].

Note that more modern psychopharmacological methods legitimized nowadays, although they do not mechanically cut nerves, can be no less detrimental for the patients as regards their health stability limits. Mechanical mallet-based prefrontal lobotomy, which is obviously far from being a precise low-demolition surgery, and pharmacotherapy of nervous conditions based on antipsychotic drugs have one thing in common: both techniques lead to undesirable side effects that are more a rule rather than an exception. Such techniques intentionally drive a person out of her/his natural stability limits [9].

Of course there exist less dramatic and more ubiquitous techniques in contemporary medicine that operate close to the organism stability limits. For example, there is a methodic of finding the "sugar curve" which is a colloquial name for a glucose tolerance curve (whose linear part is usually known as a dynamic range). A subject has to drink or to be injected a glucose-containing solution until the blood sugar concentration rises to a dangerous level: some diabetic patients, especially those suffering from DM-1 (diabetes mellitus type 1) or intermediate type, can experience very unpleasant effects, can even faint or be driven to coma, which necessitates rush recovery measures. The trouble is that such a test nominally requiring the glucose intake not exceeding $125 \mathrm{~g}$ (who really controls it?) can be indiscriminately administered, e.g., to the patients already having diabetic ketoacidosis. The beginning of adverse effects such as vertigo, blurred vision or even fainting marks the diabetic health boundary. In fact, this is in vivo experiment on an unsuspecting patient who undergoes a certain incalculable risk, although the test is generally considered completely safe in the medical circles.

Observing the dubious techniques leading to bodily harm inflicted by premeditation on a trustful patient may produce a psychological impact on medical personnel, e.g., by creating a careless, cynical atmosphere within the healthcare community or a feeling of disappointment by some part of the doctors. Would you like to visit a physician who is disappointed in the medicine?

The mHealth technologies offer a different paradigm compared to that adopted by the contemporary clinical medicine. This paradigm largely consists in monitoring the differential dynamical landscape of the functional status of an organism. Eventually, mHealth must ensure a high-resolution body mapping i.e. provide the continuous-time multi-channel registration of physiological information manifesting the organism's inner working. In the mHealth paradigm, the health margins can be measured by putting weak external signals on sensors (actuators) in the course of a person's daily activities and measuring the body response. An amplified response would testify to attaining the health domain boundary, where, e.g., a bifurcation can occur. An important thing is that one need not purposefully take a person to her/his health limits. In short, the mHealth concept enables to analyze the dynamic scenery of an organism as a working entity interacting with the environment. 


\section{Sensors}

The mHealth sensors are designed to convert the body physiological characteristics such as pulse rate, blood pressure, ECG (electrocardiogram), EEG (electroencephalogram), HEG (hemoencephalogram), REG (rheoencephalogram), PPG (photoplethysmogram), ENG (electronystagmogram) and EMG (electromyogram) signals, local thermal (infrared) emission, skin wetness, etc. into sequences of electric pulses and further into digital signals that can be processed by some central unit, at present by a computer or a smartphone. In particular, many currently available sensors measure or register local optical or electric characteristics of the human body. Electromagnetic (EM) radiation enabled sensors provide physiological information since the outgoing EM waves (wireless signals) are modulated by physiological processes. Sensors in fact eavesdrop on the body intrinsic signals (eigensignals) accompanying the functioning of the metabolic reactor known as human organism. Eventually the mHealth sensors will be capable to emulate natural body receptors.

The physical quantities registered or measured by medical sensors are typically temperature, pressure, position, acceleration (e.g., through extremely small MEMS i.e. microelectromechanical system based accelerometers), force, torque, concentration of various substances (such as oxygen or $\mathrm{CO}_{2}$ ), voltage, electrical conductivity, magnetic field strength, fluid (blood, urine) properties such as density, viscosity, dielectric constant, etc. Apart from sophisticated hardware implementation, sensors are usually designed to operate through efficient algorithms so that creating dedicated applications is an indispensable part of mHealth technology and business, specifically when one needs real time multi-parameter health monitoring. Note that there is always a working range of measured quantities and an operating temperature range, and software must ensure automatic control of these ranges.

Advanced mHealth devices must operate continuously $(24 \times 7)$ on a sufficient output signal level, which implies that small-sized and lightweight batteries should last a long time (at least $\leftrightarrow 10^{3}$ hours) without significant drop of initial voltage and output current. Note that autonomous, ultrasmall-scale and long-lasting power supply is so far a great problem. The mHealth monitoring devices are especially useful under "normal conditions" i.e. when a monitored person performs her/his daily life activities. Fluctuations and noises related to the body motion must be somehow handled or subtracted (at best in real time), which is mainly a software and algorithm design problem. The whole choreography of sensor messages must provide a coherent integral picture of the organism on the usual human time scale, exhibit logical correlations and comprehensively describe the collective, overall state of health. Sensors registering the body's eigensignals and the appropriate software comprise the foundation for a new breed of devices for continuous monitoring, non-intrusive diagnostics and nearly harmless therapy of the entire organism. One might envisage the steady development of mHealth technologies to almost total unobtrusiveness, $24 \square 7 \times 365$ non-demolition measurements of physiological parameters, lightweight and esthetic attractiveness.

It is essential that sensors enable one to register local parameters. For example, metabolic changes in some body area can result in the redistribution of subcutaneous blood flow leading to minor local temperature and skin conductivity modulations. Thus, a number of measurable parameters and conditions (local hyperthermia, variations of skin electric conductivity, electric and magnetic field distribution around the body, etc.) can serve as indicators of local metabolic processes inside an organism. Accordingly, one can establish the state of health with respect to metabolic diseases and, e.g., provide means for non-invasive monitoring. This remote monitoring resembles dynamic computer-based cartography and remote sensing widely used in applied sciences. Use of high-quality computer software is indispensable for this kind of problems due to their inverse (incorrect) character. So the health state is global (i.e. related to the whole organism) whereas its manifestations or the deviations from it may have a local character mapped by sensors.

One can again refer to the methods adopted in the oriental medicine. In particular, diagnostic techniques of oriental medicine are based on multichannel processing of physiological signals, e.g., while trying to analyze the pulse (heartbeat) rate combined with the observation of the tongue, palate, face and sclera color, listening and smelling the breath, etc. Each observation channel in oriental medicine is characterized by rather high resolution i.e. a specially trained doctor attempts to discern the tiniest details of the observed body function. Thus, when taking the pulse, she/he tries to place it under a large number (about 20) of intuitively clear and irreducible categories: uneven, choppy, empty, tight, abrupt, etc. [10]. It is interesting that the diagnostic signal is perceived by the tactile sensory receptors located on the fingertips, but is interpreted in terms of rather complex images. In a similar way, electronic or quantum sensors in mHealth can provide analogous measurements, whereby the obtained images can be rather complex, with the image categories being quantitatively delimited (by numbers) and not intuitively comprehended (by metaphors).

Notice that in mHealth different sensors located on the body can, in principle, work independently measuring diverse physiological parameters. Cross-correlation of such measurements (simultaneous or taken at shifted time instants with respect to one another) would provide interesting information about the state of an organism. The respective correlation matrix can characterize intrinsic couplings between various body subsystems. For instance, time delays between different sensor readings registered as responses to the same stimulus, e.g., pain, stress-ECG, local thermal excitation, environment temperature changes, verbal assault, alcohol, etc.) may describe differential impact of any given stimulus on the entire organism. Decay curves of separate sensor responses would provide information on the 
relaxation to the equilibrium (health) state i.e. a quantitative measure of curability. Of course, all such data would be very hard to obtain and analyze without dedicated software.

Sensors can be wearable (also placed in body cavities) or implantable. Implantable sensors are the most complicated since they must be very small and light-weight on the body scale and consume very low power. Besides, they require a surgeon to implant them, special licensing and must not deteriorate with time. It is essential that stand-alone sensors basically provide local information, in distinction to sensor networks to be discussed in the next section. As already mentioned, such information, nonetheless, can be very valuable. Thus, sensors registering, e.g., small local variations of the body temperature, perspiration, electrical conductivity of the skin and, accordingly, local changes of electric field distribution around the body can provide data which, if properly interpreted, would map, in particular, diabetic disturbances in various subsystems of the organism.

Recall that all sensor data contain uncertainties. The reason for this is that one cannot control all the factors that influence a specific measurement. Uncertainties are inherent in any recorded value which is inevitably restricted by finite accuracy, no matter how refined the measurement procedure could be. Note that measurement errors are not mistakes, but a natural consequence either of incomplete knowledge or of time variations (e.g., drifts due to dynamic instabilities). The uncertainty of a measurement is characterized by a \pm range (error bars) that are in general non-symmetric. The uncertainty range can be elucidated by making repeatable measurements which is, however, seldom possible in the mHealth environment. For most people who have not been directly involved into engineering or scientific activities, the concept of measurement errors is usually hard to grasp: one tends to take the measured parameters (e.g., temperature, arterial pressure, pulse rate, ECG signal amplitude, concentration of chemical substances, etc.) as true and final values which they are not.

In order to reduce uncertainties in mHealth one has to separate random errors from biases, e.g., through performing repeated measurements at the initial (pre-deployment) stage of engineering implementation. One can see that there exist a number of conflicting requirements that should be balanced when designing a working mHealth system. These engineering conflicts are drastically aggravated when one tries to unite sensors into networks with wireless interconnection between the nodes.

\section{Body Networking}

The grid of miniature interconnected devices, mostly sensors that can be wearable or implantable, is usually known as a body area network (BAN). Uniting separate sensors into networks, e.g. BANs, entails specific networking problems (scalability, latency, reliability, responsiveness to topology changes, network overhead, computationally expensive security implementations and so on). The most convenient networking connectivity appears to be via wireless channels, the respective networks are known as wireless body-area networks (WBAN) and wireless sensor networks (WSN), see an overview [11]. In the case of massive deployment of body networks, additional problems may arise such as interference and electromagnetic compatibility (EMC). In particular, choosing right frequencies can be an obstacle for the massive WBAN/WSN deployment since the EMC requirements and frequency allocation procedures vary from country to country and can be very cumbersome. Note that the issue of serious electromagnetic health impact is usually not raised for the power levels at which WBAN/WSN typically operate. Moreover, sensor networks for mHealth are by default mobile so that the network should be capable to deal with variable data links and movable nodes. Physically, it means that the mobile network output signals should be invariant under the changed state of motion. In short, to design a robust mobile WBAN/WSN containing many nodes can be a challenging task, which is aggravated by the power efficiency requirements. In particular, one can hardly imagine that elderly patients would be enthusiastic about the necessity to charge or replace the batteries every other day.

When the nodes of a body network are placed very densely, the network is capable to register a nearly continuous spread of human body parameters - the "field". In the limit of very small (in continuous mathematical models, infinitesimal) spacing between the nodes this "health field" would be represented by a spatio-temporal distribution of physiological variables describing the functional phase of the body at a given instant of time. Such continuous limits may appear unrealistic yet they are indispensable tools in any exact science. It is of major importance that the distributed field of body parameters consists of a set of directly measurable quantities, each of them can be characterized by a numerical value and measurement error. Thus, the necessity to introduce purely hypothetical notions allegedly characterizing the organism status (such as bioenergy, channels, waves of bioinformation, extrasensorics, aura, etc.) is removed and the unobservable notions themselves become superfluous. In other words, Mobile Health leads to Measurable Health.

Sensor-based mHealth networks can in fact supplement the human sensory system that had been developed as a survival mechanism of human species. Sensory signals in highly complex organisms such as humans are always related to biofeedbacks. Any biofeedback device must pick up information of the current state of a human and provide it to sensory mechanisms. For example, visual sensors help us to coordinate movements, drive a car, protect ourselves, etc. Other natural body sensors, e.g. reacting on the rising skin temperature, allow one to evade burns and so on. Artificial sensors - wearable or implantable - can amplify weak and not immediately noticeable signals which, however, manifest some irregularities or health anomalies. For example, stress-related extrasystoles (mainly designated as PVCs - premature ventricular contractions or PAC - 
premature atrial contractions) are usually unnoticed by a person and considered a benign condition by most doctors. Nevertheless, extrasystoles can be a manifestation of a severe, though perhaps asymptomatic or not clearly diagnosed, pathological condition such as coronary artery disease (CAD) leading to acute myocardial infarction or even sudden cardiac arrest. Thus, paying attention to latent symptoms that can only be captured by sensitive artificial sensors may be vitally important - this is the essence of mHealth approach. Eventually, as already mentioned, the mHealth sensor-based body networking must emulate the body own sensory system (recall that, e.g., the human skin is full of sensory receptors, see, e.g., [12]), with the advantage of providing long-distance biofeedbacks. The crucial difference between the networked signaling pathways in a functioning organism and the man-made body sensor networks is that most of the biological software controlling the organism's own sensors is encoded in the DNA and the "hardware" is mainly implemented via synaptic computation, while the mHealth sensor technology is controlled by human-produced codes. Accordingly, the complexity of biological sensor networks is many orders of magnitude higher than anything created by humans. As coding and sensor technology improve, artificial body networks will tend to natural ones (mostly studied in neurophysiology).

Notice that biofeedback can be amplified by using either special sensor-based devices [13] or special techniques. It seems that yoga was engaged during many centuries in empirical development of biofeedback techniques [14]. One of the most common biofeedback devices is the usual mirror; it provides us with the reflection of ourselves and hence the biofeedback information for better control over our behavior and better movement accuracy (e.g., when one is combing hair or shaving oneself). It is not a matter of mere convenience, but a clever use of biofeedback that most repetition studios for ballet dancers and some martial arts training halls are equipped with mirrors. Muscular activity relying on sensory mechanisms and based on biofeedback is a salient example of a synergic functioning of different body subsystems such as the brain, the autonomic nervous system and sensory organs.

The fabric of devices placed on the human body can of course consist not only of passive or semi-passive (registering and transmitting) sensors; there may also be actuators introduced into the body grid that would be able to transform external signals into actions, thus influencing or even controlling the body functionality. The fast growing field of quantum-enabled technologies (in particular, nanotechnologies) being more and more transferred from R\&D to the industry now lead to the manufacturing of miniature interconnected devices that eventually can talk to one another. A network of quantum-enabled devices ensuring mobility and wireless connectivity would be highly adaptive, operate on low power levels and would not require much human attention or maintenance. As the number of interconnected devices surpasses the global population ( $\leftrightarrow 10^{10}$ nodes), one can envisage, figuratively speaking, the fully developed Internet of Things.

The mHealth solutions essentially perform a duplex radio communication, when sensors convert the data collected from the body into properly modulated signals, receive and transmit them using tiny antennas. One can regard the sensor-based mHealth wireless networks as proxies for the natural body sensor systems. A wireless router or a smartphone nearby takes these signals, decodes them if necessary and forwards the information to the Internet or via a private network using, e.g., an Ethernet connection. This procedure can be of course reversed, the router receiving information from the Internet, converting it into modulated signals and re-sending them to body sensors or actuators. The whole two-way transmission process, though looking conceptually simple, is in fact computationally-intensive requiring highly reliable data transmission or exchange, fast data delivery (high temporal resolution), each node mobility support, real-time computation of the nodes' actual positions and so forth. Furthermore, the network must communicate with the back-end server database containing confidential health data for each user. Authorized clients can access this data after fast and secure authentication procedure, for example, the personal physiological information can be analyzed offline by a family doctor. Design of appropriate middleware presents a separate problem [15] to be possibly handled by large software companies such as Microsoft.

The main purpose of WBAN/WSN consisting of a discrete set of interconnected nodes is to collect the body data and transmit it wirelessly to a base station and further to a remote location to be processed there offline or online, with recommendations, warning or alarm being sent back. The term "base station" should not be confusing in the mHealth context: it is in fact any local processing device used to receive and aggregate data from WBAN/WSN; this device can be a smartphone, a notebook, a PDA, etc. The base station operates as a router between sensor nodes and a remote server using wireless channels. Notice some significant differences between general purpose sensor networks (e.g., used for environmental monitoring) and WBAN/WSN used in mHealth. In the mHealth case mobility is the crucial requirement whereas the general-purpose WSN can consist of distributed fixed-position nodes over some terrain. Moreover, WBAN/WSN in mHealth must typically ensure higher data transmission speed due to, e.g., increased sampling rate in physiological measurements such as ECG, EEG, arterial blood pressure monitoring, etc. (often resulting in so-called Big Data) and admit frequent manipulation by humans.

\section{Wireless Technologies for Healthcare}

Sensor-based wireless body-area networks (WBAN) and wireless sensor networks (WSN) can be, in principle, organized as personal-area networks (PAN) or even as standard interconnected local area networks (LAN) relying on messages that move blocks of bytes (data packets) from 
the body to data collecting structures. However, strict layering for WBAN/WSN does not seem to be present yet, and it is primarily the task of IEEE (more specifically, EMBS - Engineering in Medicine and Biology Society) in cooperation with the leading ICT companies to define how to appropriately format WBAN/WSN messages. There are still open engineering questions, in particular regarding the responses to WBAN/WSN messages, properly identifying message senders, arranging source and destination information, encoding, etc. One may note that messages sent by body sensors can be mission-critical and require human reviewing before forwarding further so that the possibility should be envisaged that the transferred block of bytes could be converted into human-readable data structures at least at certain stages.

All wearable or implantable body sensors can address the same router to get connected to the Internet so long as they have wireless capabilities, e.g., an amplifier and an antenna. Routing all the sensors through IP in effect implies a single network, which is important from the viewpoint of servicing and upgrading. However, if the sensor network is very dense and, for example, all the sensors are working simultaneously, the bandwidth can be exhausted so that interference effects may be observed or connection can be lost. The latter is obviously unacceptable for mission-critical applications that are encountered in mHealth or telemedicine. What is more, higher operation frequency range needed to transmit more data usually requires more power which can be a stringent limitation for sensor-based body networks.

Although the substance of health issues, in particular, recommendations received through the mHealth system commonly have a clear priority over the security problems, users (e.g., patients) should not be carefree about security since attacks with the use of wireless data communication protocols (such as Bluetooth, Wi-Fi, ZigBee, UWB, NFC, etc.) can impair the operations of an mHealth system. In general, deploying new technologies such as mHealth without due regard to security can compromise a consumer's privacy since wireless networks are by their physical nature very vulnerable unless special security measures are taken. Besides trivial squattering or piggybacking, there exist a long list of disruptive activities that can cause much harm to mHealth users [16]. For instance, a person can lose her/his job or be deprived of insurance protection if a leakage of sensitive physiological data occurs. What is more, life-threatening conditions may arise, e.g., a wireless-controlled insulin pump can increase or decrease the insulin output to potentially dangerous level, this change of mode being noticed neither by the patient nor by the doctor. Wireless cardiostimulators or defibrillators can also be vulnerable to malicious attacks. Unfair employers, political rivals, competitors and other potential adversaries might use social networks (such as Facebook) to reveal embarrassing personal health information obtained through mHealth security flaws.

Although currently available wireless protection keys based on WPA/WPA2 standards (Wi-Fi Protected Access) with AES (advanced encryption standard) or TKIP (temporal key integrity protocol) specifications are considered to prevent security flaws and, moreover, are supported by most routers, they can still be hacked, e.g. by brute force or with the help of dedicated software. Both AES and TKIP encryptions are implemented to prevent the wireless signal from being intercepted and decoded. Previously common wireless encryption protocol (WEP) also known as "wired equivalent privacy" was cracked many times with the help of software using some search algorithms mainly because WEP is a fixed key (that is the main reason why WPA/WPA2 standards have replaced WEP: they have variable keys that are changed after specified time intervals).

Recall that any Wi-Fi device has its own MAC-address (media access control - a 48-bit number usually written as a series of 6 hexadecimal octets) that is assigned by the manufacturer. One can regard MAC-address as a signature of each networked device. Yet one can overwrite (more exactly, override) or bypass the MAC-address through the procedure known as MAC spoofing, especially in Open Source systems. For example, Linux contains a program called macchanger giving to the user a priority over factory-burned MAC-address. Another way to change MAC-address in Linux is to use script commands ("ifconfig" etc.). In short, changing MAC address is much easier in Open Source than in Windows systems.

One might think that security issues play an inferior role compared to much more substantial health matters. This is generally true, but leakage or disruption of wirelessly transmitted sensitive health data combined with ever increasing frequency of Internet attacks can so strongly affect a person's life that one should take the security problem quite seriously. In order to prevent compromising wireless body networks and inflicting danger to the user's health one ought to consider possible security and privacy loopholes already at the application design stage and not postpone their elimination for future work as is currently the case. One can find a rather comprehensive overview of security issues related to mHealth in [17].

It may be pertinent to say a few words about the protocols and standards currently applied for wireless communication. Since wireless networking is a rapidly developing field there can be a baffling number of available protocols and standards, many of those still incomplete. Thus, one would need first to figure out what is the difference between them and what are the criteria for choosing the most suitable for mHealth.

The mHealth technology must sometime "break out of its shell". One of the main challenges in mHealth lies in the absence of standards or in incomplete standards. This drawback specifically concerns WBAN/WSN management, interoperability, reliability and security. For example, in the sensor-smartphone connectivity there are at least four competing specifications: Wi-Fi, Bluetooth, NFC and ZigBee. The choice between the Bluetooth protocol and others is especially critical since Bluetooth is mostly used to connect the body sensors with the mobile base station, e.g., 
smartphone or notebook. Bluetooth is a short-range wireless standard designed for mobile devices with the primary intention to replace highly unreliable infrared link and mainly applicable in personal area networks (PAN). What is known as the Wi-Fi set of protocols (IEEE 802.11), they in general enabled the ubiquitous use of the Internet which has become as commonplace as cellular phone coverage. Wi-Fi has been designed not necessarily for mobile applications; it is just a wireless option for LAN (Ethernet networks) and can be considered a generic term designating an alternative to cable networking rather than a single concrete specification.

Although there exist many similarities between Bluetooth and Zigbee, for instance, they both work around $2.4 \mathrm{GHz}$ and belong to the same family of wireless specifications IEEE 802.15 , the two standards are different as regards their possible application as well as the devices they have been designed to network. While Bluetooth has been oriented at replacing cabling and infrared links between personal devices (originally, between cellular phones, PDAs and notebooks), Zigbee has been intended to provide connectivity between home appliances such as lighting or heating devices, air conditioners and the like. Accordingly, high data rates were not indispensable for Zigbee - in distinction to Bluetooth which was used for document exchange. Accordingly, Bluetooth can move larger data packets. This difference between the two standards is counterbalanced with lower power consumption by Zigbee devices so that, e.g., Zigbee-connected sensors can last longer. Moreover, Zigbee tends to operate on longer distances and can thus interconnect more devices than Bluetooth. Yet this difference typically becomes inessential for body-area networks. What seems to matter is a relatively large delay in joining the Bluetooth network (up to several seconds) which can be unacceptable for mission-critical applications encountered in medicine.

To provide wireless services for the long-haul mHealth arm (backhaul) WiMAX (worldwide interoperability for microwave access) communications standard IEEE $802.16 \mathrm{e} / \mathrm{m}-2011$ can be used. WiMAX is mainly adapted for metropolitan networks (MAN) i.e. providing wireless Internet access across city area. However, WiMAX requires special infrastructure such as transmitting towers, big receivers, trained personnel, etc. and can, as a rule, be operated through the state or municipal agencies. WiMAX has been designed to combine wireless and broadband so that WiMAX can provide long-haul high-speed connectivity enabling the mHealth wireless access to large areas (WAN). Yet there is an optimal distance after exceeding which the bit error tends to increase [18]. One can, nevertheless, envisage that WiMAX technology for mHealth will rapidly develop, in particular, endowed with MIMO (multiple input multiple output) antenna implementations.

The question with mHealth networks is: how to achieve the balance between interoperability, mobility, reliability, scalability, power efficiency and sustainable connectivity without bringing additional (and unnecessary) complexity and sacrificing ease of use. Although the underlying technology can be rather intricate, its users - both general consumers and medical professionals - should not notice it. Moreover, maintenance of mHealth customer mobile equipment should be at least as unobtrusive as of modern phone subscriber lines. Lack of permanent seamless communication throughout personal mHealth systems would drastically diminish their market acceptance.

\section{Market Acceptance and Response}

Medical doctors have traditionally been reluctant to use advanced means of communications, now incorporated under the umbrella of mHealth, on understandable reasons: firstly, they do not want to invest extra time in learning; secondly, they perceive their actual workload as exceedingly high; thirdly, they may feel a danger of being subjected to new legal liabilities.

The doctors would typically prefer to deal with a passive patient who meekly accepts any recommendation of the physician. Challenging or questioning the doctor's judgment has traditionally been out of the question. The mHealth patient might be quite the opposite: she/he tends to be prepared to the contact with a doctor and will provide a list of questions, expecting to be answered in clear terms. A mHealth patient has a free access to her/his health records and can share these data through the Internet to quickly receive a set of "second opinions". Already this easiness of communication can make diagnoses and decisions about possible treatment more accurate, minimizing hand-waving, perfunctory manner in which such diagnoses and decisions are often made today. Moreover, it would be more difficult for a doctor not to listen or to interrupt a patient - a common practice in today's medicine. It would not even involve extra time or costs to get better outcomes for the patients: only a slight change of attitudes induced by technology.

Having a set of second opinions is particularly important when a potentially life threatening condition is suspected. However, the query for a second opinion may disturb the patient-doctor relationship as perceived by all parties concerned: the doctor may feel hurt interpreting the request for a second opinion as the manifestation of distrust or questioning the doctor's competence; the patient may be too embarrassed or shy to ask his physician for a rival opinion.

The mHealth can be projected to shift the distribution of the whole massive of patients with respect to their attitudes toward healthcare between two extremes "I want the doctor to decide for me" and "I want to decide for myself, just give me all the facts" in the direction of the latter choice. This can lead to an important transformation in the patient-doctor relationship. For any organism's instability manifested as some pathological process, one can probably find an optimum, when decisions are made jointly by a patient and a doctor (an interpolated decision between the two extremes).

An important factor - as always - is time: one can get the relevant information about one's health (in particular, as a second opinion) almost instantly whereas obtaining a 
judgment from a medical doctor is usually a tedious procedure that may last a few weeks. Besides, economic factor is important: high costs associated with delivering healthcare i.e. through a personal contact with a doctor (honoraria, fees, etc.) are drastically reduced. Yet as far as the "second opinion" goes, not only the doctors, but also most external parties such as insurance companies, governmental organizations and other outside payers tend to regard the quest for a second opinion as an unnecessary parallelism, waste of time, money and other resources.

According to the comprehensive "Aging World" report [19], the percentage of older people (over 65) is already of the order of 10 percent of the total population and is projected to reach $14-15$ percent by $2040-2050$ comprising about 1.4-1.5 billion people. If aging people have to or prefer to live alone, they would need continuous and inexpensive health monitoring without disturbing the life-style. In this context, the emerging mHealth technology can potentially transform the way of life making people healthier while maintaining the required level of comfort [20]. No surprise that according to a research from Frost \& Sullivan [21] the mobile health (mHealth) applications market will see revenues grow from $\$ 230$ million in 2010 to about $\$ 400$ million in 2015. Here, one should distinguish between healthcare and homecare since the first is mainly focused on preventive measures and health monitoring whereas the second does not exclude radical medical treatment.

Notice that the abovementioned body physiological characteristics presumably taken up by the sensors are not always directly measurable physical quantities such as position, length (or its variations), frequency, voltage, electrical conductivity, pressure, etc. This fact, though often overlooked by non-specialists, is very important for assessing the accuracy of measurements and hence the reliability of non-invasive sensor-based mHealth monitoring. For instance, the market failure of formally very attractive non-invasive optical glucose sensor options seems to be the consequence of low accuracy of these techniques compared to those based on the traditional finger piercing blood probes. Portable blood pressure monitors suffered the analogous fate: no reliable and miniature blood pressure monitors seem to be commercially available yet on a wide scale, at least such devices that would not perturb normal life activities. Moreover, the whole mHealth system must be comfortable to wear, maybe even attractive and fashionable like a wrist watch or embellishment.

To acquire wide market acceptance and success special applications and services should be provided for WBAN/WSN systems. Such applications must get information from multiple nodes, and a user (e.g., a medical doctor) must be capable to view both the aggregated and piecewise information upon a simple request. Health-relevant data can be represented in versatile forms: text, $2 \mathrm{~d} / 3 \mathrm{~d}$ graphics, video, multimedia, enhanced reality; such data may be structured or unstructured and so forth. Streaming data can require not more than $10^{-3}-1$ second to invoke an automatic response. Software analytic toolkits may be necessary to interpret unstructured data and support understanding of health/medical conditions. Of course such trivial applications as appointment reminder, event journal, health deterioration alerts, automatic emergency signals can be included by default in the mHealth software design.

Notice that the innate property of mHealth systems consisting in by default automatic data documentation is of course beneficial for the patient, but can be met with mild rejection by the medicals. It would be also pertinent to note that mHealth solutions can contribute not only into the health of a person who is directly using them, but also in the safety of other people. For instance, a person must always be fit when driving a car, yet today this fitness cannot be checked in real time. Many accidents occur as a consequence of a sudden heart attack, stroke or epileptic seizures (see, e.g., [22]). It is a difficult situation also from a legal standpoint because the person who caused an accident (possibly with injuries and deaths) could not foresee a sudden deterioration of her/his health conditions. One can, however, use sensor-based mHealth systems to prevent such events. Sensors (WBAN/WSN) would produce an alarm signal to the driver and/or to the car as soon as at least one of the health parameters hits the dangerous zone near the health domain borders. This real-time control can become ubiquitous in any professional environment associated with the risk involving other persons, e.g., for bus drivers, train conductors, ship captains, aircraft pilots, plant operators, surgeons, etc.

As already mentioned, one of the contretemps for fast development of mHealth systems may be associated with the reluctance of medical professional to adapt their working habits to radically new technologies. More exactly, the doctors and medical personal will be using mHealth devices and related procedures only as long as they would feel that obvious convenience can be provided by such systems. Market admittance may also be impeded by the necessity to retrain clinicians, physicians, medical technicians and the entire medical staff involved in doctor-patient relationship (including high-positioned doctors) on a large scale. Most medical doctors are, according to numerous surveys (conducted, e.g., in Germany), generally content with their work, but often complain about work overload and fatigue. In this respect, mHealth solutions can provide a relief of the strain, freeing more time for each individual patient.

\section{Case Studies}

A prototypic approach to mHealth solutions was exercised already in early 1980s by "Biosig Instruments" [13], a Canadian company designing and manufacturing electrophysiological fitness systems. This company has developed a number of devices performing sensor measurements of the body health parameters. Such measurements are performed using clever electronic balancing schemes to reduce errors. Efficient algorithms are employed, in particular, to register instant pulse rate, 
quantify stress level and provide telemetry. For example, the "Biosig" engineers measure stress by linking its level to EMG (electromyographic) activity of forehead i.e. of the frontalis muscle as well as facial and neck muscles, if necessary. The measurement technique is based on empirical data about the unique relationship between the electric signals generated in neurons that govern the frontalis muscular tonus and stress as a perceived state. As a possible biofeedback channel, "Biosig Instruments" used human hearing system, a relatively slow $(\sim 10 \mathrm{Kbit} / \mathrm{s})$ but efficient data link. Moreover, there is a gamma of health control devices based on the use of sensors, biofeedback, proprietary algorithms and software developed by the same company and destined to control the health state - not necessarily to record disease-related parameters. The latter parameters can be registered as a natural continuation of the health measurements, e.g., arrhythmias and ECG readouts can be taken through simple technologies without the use of common 12-lead medical devices that can only be used in hospitals, cardiology clinics and, sometimes, in physicians' offices.

Although mHealth is a developing technology and its standards are far from being complete, many enterprises and research institutions are using currently available toolsets to create advanced mHealth solutions. The following examples demonstrate mHealth solutions that efficiently use today's technologies. These case studies may serve as models reflecting the trend to obtain multichannel body information through mHealth systems. The advancement of miniature sensors in combination with the popular consumer devices such as smartphones, tablets, netbooks, etc. makes current personal health management more and more affordable.

For example, the standard bulky cardiomonitoring devices typically containing multiple leads, to be only used in clinics or practices and hard to wear, can be transformed into tiny plastic patches that would be attached to dry skin without using a conducting gel to improve the ECG signal (note that the heart's electric signal is rather weak, ranging from 0.1 to $3.0 \mathrm{mV}$ ). The gel, by the way, usually contains propylene glycol that can cause allergies. With small, almost invisible antennas the patches can be connected to mobile devices (in particular, smartphones) so that the signal, e.g. indicating the heart fibrillation, can be transmitted to a medical practitioner or to other relevant persons. Recall that among all chronic conditions, heart failure (CVD - cardiovascular disease) is the primary killer in Europe, especially with the population getting older, urban and professional stress getting stronger. CVD is currently projected to remain the single leading cause of death: thus, according to the WHO report," By 2030, almost 23.6 million people will die from CVDs, mainly from heart disease and stroke" [23].

Another area where mHealth is advancing is diabetes management. According to the WHO statistics report, released on May 16, 2012, diabetes is one of the fastest growing problems of noncommunicable diseases. One in 10 adults has diabetes mellitus (DM) [23]. Diabetes is one of the major risk factors that entail serious side health conditions such as hypertension, CVD, kidney failure, deteriorated vision (retinopathy) and many others. Diabetes is a highly complex metabolic disorder and would require a special description and modeling, in particular, in terms of the mHealth approach. There are a number of local metabolic manifestations that can be used to quantitatively diagnose $\mathrm{DM}$, for example, by measuring glucose concentration in any body fluid. Commonly, blood sugar level is tested by taking blood samples from a patient, the least invasive method being finger piercing. Thus, the patients who suffer from DM 1 (type 1 diabetes, juvenile diabetes) have to take blood several times a day which is a painful procedure, especially for children (who constitute the majority among the DM 1 patients). A non-invasive solution was developed, in particular, by Microsoft Research in collaboration with the University of Washington, which is just a contact lens capable of monitoring the glucose level in addition to the regular vision-correcting function [24]. This solution represents a trend in mHealth technology known as the natural user interface (NUI). Multifunctional contact lenses are made of special plastic and are equipped with small built-in wireless transmitters/receivers feeding tiny antennas. The device is clearly an interdisciplinary product resulting from the cooperative effort of the specialists who represent diverse areas of science and engineering: electrical and radio engineers, experts on sensors, antenna designers, material scientists, computer specialists and ophthalmologists. The collaborative team reportedly has plans to emphasize the feedback and software components providing alerts (when the glucose concentration leaps outside the health margins) in the lens user's view field, which is a kind of enhanced reality solution.

Although mHealth is a developing technology and its standards are far from being complete, many enterprises and research institutions are using currently available toolsets to create advanced mHealth solutions. For example, a team from the University of Berkeley in cooperation with MS Research has developed a simple but neat device by combining a phone camera and a conventional microscope [17]. More specifically, this tabletop-mounted device called CellScope (Figure 1) coupled a $40 \times$ lens system with a 3-megapixel camera phone. The whole two-piece set was actually turned into a mobile laboratory capable of transmitting images from any remote location. The new device has already been successfully used in Africa to identify blood samples for tuberculosis and malaria.

The traditional way of diabetic treatment implies various combinations of short and long term insulin pumping, often alongside with other medications such as beta blockers, ACE (angiotensin converting enzyme) inhibitors, ARBs (angiotensin-II receptor blockers) and other types of antihypertensives in the case of accompanying high blood pressure. Notice that all such substances acting together can produce a strong negative impact on the patient's health (for example, there is not enough evidence on the insulin injection effect on the natural function of the pancreas). Some beta blockers may also be contraindicated for the 
patients with sinus bradycardia and partial AV (atrioventricular) block. Therefore, constant monitoring of diabetic patients is necessary to tune treatment to the continuously changing health conditions of a person.

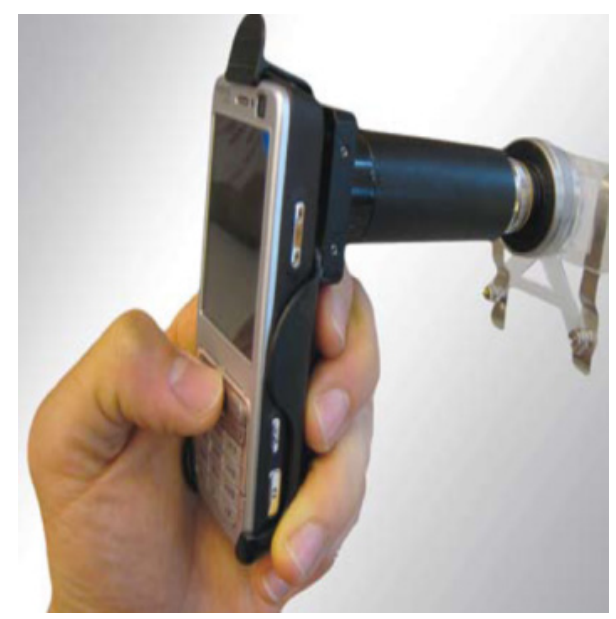

Figure 1. Cell phone microscope designed in Prof. Fletcher's Lab (Bioengineering Department) at the University of California Berkeley. The device efficiently exploits the fact that although the access to diagnostic facilities is very limited in many developing countries, access to wireless networks is almost ubiquitous. CellScope enables both visualization and transmission of microscopic images for remote diagnostics and feedback. (Source: Prof. Fletcher's presentation "CellScope: Mobile Microscopy", )

There are currently a number of mHealth solutions available on the market that are providing a direct and simple communication between the doctor and the patient, allowing one to monitor the reaction on medication in the $24 \times 7 \times 365$ mode. For example, the University College of London has developed a technology using standard tools such as Microsoft Dynamics CRM client mobile access (PDA, smartphone, etc.) to connect both the families and the medicals in order to observe the prescribed treatments and medication scheduling as well as to provide timely feedback involving the clinician (e.g., to inform on negative side effects of medication, increase or decrease of the blood sugar level as a response to treatment, etc.).

\section{Conclusion}

Although real-time wireless mHealth applications are facing many challenges, the sensor-based network systems are capable, as already mentioned, to establish individual health margins, thus paving the way to the ultimate customization of handling body problems. For instance, each prescribed drug or physiotherapeutical procedure can be tested, through harmonizing a person's stability margins with the available data on the impact of pharmacological substances or physical agents in real time (or near real time) against individually manifested adverse effects. Both currently available Web services and Web-based applications to be specially developed can be used for this verification procedure.

One can see that mHealth is a salient example of interdisciplinary effort: to be active in this field one has to deal with sensors, networks, wireless technologies, software development and engineering (in particular, code writing and adaptation), electrophysiology, metrology and possibly a number of other disciplines. Currently, there does not seem to be off-the-shelf solutions for mHealth so that the looming disproportionate media hype is not justified at all. One might also notice that the described approach - to find first the state of equilibrium characterized by a domain of a system's parameters within the health margins and then deviations from such a state i.e. instabilities leading to pathological conditions or diseases - is universally adopted in a number of engineering and scientific disciplines, but does not seem to be popular in medicine yet. However, this is a ubiquitous problem: the lack of knowledge about the health status (i.e. the equilibrium state of human organism) and about the limits of health domain in the space of physiological variables (analogous to the space manifold in natural sciences) manifests a more general situation than currently observed in medical disciplines. The study of complex macroscopic systems interacting with the environment (e.g., social, economic, ecological, global energy, climate, etc.) is still at its infancy.

One should not consider the concept of physiological phase manifold i.e. the space of all physiologically relevant variables a superfluous abstraction. This concept proved its usefulness in all physical and engineering sciences and can be helpful to solve very concrete and urgent problems. In particular, extracting some key variables from the entire physiological phase space - mathematically this procedure amounts to a projection - one can computer-analyze the data dynamics in the obtained submanifold and make intelligent predictions. For example, forecasts of hospital readmissions have been performed by using a subset of 40 physiologically relevant variables, which allows the clinicians to better assess risks and avoid penalties [26]. One can experiment with other physiological subspaces to increase the accuracy of prognoses and to obtain new results.

Note that one can apply mHealth solutions to control or monitor drug and alcohol abuse, the fight which is hopelessly lost by both healthcare workers and governments. In particular, mHealth technologies implemented through wireless sensor networks (WBAN/WSN) are capable of carrying out differential diagnostics of stress and of other conditions induced by chemical agents. Apart from passive detection, biofeedback mechanisms can be utilized for the reinforced correction of behavior and to check up for relapses.

It is interesting that mHealth solutions due to their mapping functions can empirically disprove (or confirm) not validated so far healing concepts such as those based on so-called extrasensory perception(ESP), bioenergy, auras, chakras, qi, meridians, acupuncture, miraculous icons, etc. [27]. Although quite popular in the lay milieu, these concepts are not shared by the contemporary evidence-based medicine and therefore currently have the status of pseudoscience [4]. However, it may well be that each inner body organ has its 
representation area on the skin surface so that one can stimulate such mapped active zones to exert an inverse influence on inner organs (tail-wagging-the-dog principle). In any case, one can employ mHealth solutions to provide an empirical meaning to currently unverified concepts and to get rid of numerous speculative notions, when an illusion of the understanding of new phenomena is achieved by inventing new names for them.

\section{REFERENCES}

[1] K.A. Johnson, J.R. Svirbely, M.G. Sriram, J.W. Smith, G. Kantor, J.R. Rodriguez. Automated medical algorithms: issues for medical errors. J. Am. Med. Inform. Assoc., Vol.9, 56-57, 2002.

[2] The Medical Algorithm Project. Online available from http://medal.org.

[3] D. Luchins. Two approaches to improving mental health: positivist/quantitative versus skill-based/qualitative. Perspectives in Biology and Medicine, Vol. 55, No.3, 409-434, 2012.

[4] S. Singh, E. Edzard. Trick or Treatment. W.W. Norton, New York, 2008.

[5] US apologizes for deliberate damage. Online available from http://www.livescience.com/health/etc/101001-apologize-for -infecting-guatemalans-with-stds-years-ago.html.

[6] Surgical experiments on slaves. Online available from http://www.medscape.com/viewarticle/479892.

[7] Lobotomy. Online available from http://en.wikipedia.org/wi ki/Loboto-my.

[8] J. Engel, Jr., M.D., Ph.D. A Randomized, Control Trial of Epilepsy Surgery. New England J. Med., Vol. 345, 365-367, 2001.

[9] J.D. Pressman. Last Resort: Psychosurgery and the Limits of Medicine. Cambridge University Press, Cambridge, U.K., 1998.

[10] Diagnostic Methods. Online available from http://www.orientalmedicine.com/diagnostic-methods.

[11] B.J.G. Ko, C. Lu, M.B. Srivastava, J.A. Stankovic, A. Terzis, M. Welsh. Wireless sensor network for healthcare. Proc. IEEE,Vol.98,1947-1960, 2010.

[12] Receptor. The Free Dictionary. Online available from $\mathrm{http}: / /$ medical-dictionary.thefreedictionary.com/Cutaneous $+\mathrm{r}$ eceptor.

[13] Biosig Instruments, Inc. Online available from http://www.biosiginstruments.com/.

[14] R. Manocha, Researching Meditation. Online available from http://www.researchingmeditation.org/blog/category/meditat ion/sahaja-yoga-meditation.

[15] A.B. Waluyo, I. Pek, X. Chen, W.-S. Yeoh. Design and evaluation of lightweight middleware for personal wireless body area network. Pers. Ubiquit. Comput., Vol. 13, 509-525, 2009.

[16] J.H. Pardue, P. Patidar. Threats to healthcare data: a threat tree for risk assessment. Issues in Information Systems, Vol. 12, No.1,106-113, 2011.

[17] P. Kumar, H.-J. Lee. Security issues in healthcare applications using wireless medical sensor networks: a survey. Sensors, Vol. 12, 55-91, 2012.

[18] Mobile WiMAX throughput measurements. Online available from http://www.wimax.com/ and http://www2.rohde-schwa rz.com/file_16690/1SP10_2e.pdf.

[19] An Aging World. Online available from http://www.census.gov/prod/2009pubs/p95-09-1.pdf.

[20] S. Koch, M. Hägglund. Health informatics and the delivery of care to older people. Maturitas, Vol. 63, 195-199, 2009.

[21] Frost and Sullivan research. Online available from http://www.prnewswire.com/news-releases/frost--sullivan-w hats-next-for-mobile-health-157181195.html.

[22] Epilepsy and driving. Online available from http://en.wikipedia.org/wiki/Epilepsy_and_driving.

[23] World Health Organization Europe. Online available from http://www.euro.who.int/en/what-we-do/health-topics/nonco mmunicable-diseases/obesity/news/news/2012/5/world-healt h-statistics-2012-report-increase-of-hypertension-and-diabet es

[24] A contact lens diabetic monitoring. Online available from http://research.microsoft.com/en-us/collaboration/stories/nui _contactlens_cs.pdf.

[25] Attachment turns mobile phone into microscope. Computer, Vol.42, No.12, 23-25, 2009. Online available from http://research.microsoft.com/enus/collaboration/focus/health/cellscope.pdf.

[26] Predixion Software. Online available from https://www.predixionsoftware.com/

[27] US National Center for Complementary and Alternative Medicine (NCCAM). Online available from http://nccam.nih.gov/. 